Kragujevac Journal of Mathematics

Volume 45(1) (2021), Pages 7-20.

\title{
SANDWICH THEOREMS FOR MULTIVALENT ANALYTIC FUNCTIONS ASSOCIATED WITH DIFFERENTIAL OPERATOR
}

\author{
ABBAS KAREEM WANAS ${ }^{1}$ AND ALB LUPAŞ ALINA ${ }^{2}$
}

\begin{abstract}
The purpose of this paper is to derive subordination and superordination results involving differential operator for multivalent analytic functions in the open unit disk. These results are applied to obtain sandwich results. Our results extend corresponding previously known results.
\end{abstract}

\section{Introduction AND Preliminaries}

Let $H=H(U)$ denote the class of analytic functions in the open unit disk $U=$ $\{z \in \mathbb{C}:|z|<1\}$ and let $H[a, p]$ be the subclass of $H$ consisting of functions of the form:

$$
f(z)=a+a_{p} z^{p}+a_{p+1} z^{p+1}+\cdots, \quad a \in \mathbb{C}, p \in \mathbb{N}=\{1,2, \ldots\} .
$$

Also, let $A_{p}$ be the subclass of $H$ consisting of functions of the form:

$$
f(z)=z^{p}+\sum_{k=p+1}^{\infty} a_{k} z^{k}, \quad p \in \mathbb{N} .
$$

Let $f, g \in H$. The function $f$ is said to be subordinate to $g$, or $g$ is said to be superordinate to $f$, if there exists a Schwarz function $w$ analytic in $U$ with $w(0)=0$ and $|w(z)|<1, z \in U$, such that $f(z)=g(w(z))$. This subordination is denoted by $f \prec g$ or $f(z) \prec g(z), z \in U$. It is well known that, if the function $g$ is univalent in $U$, then $f \prec g$ if and only if $f(0)=g(0)$ and $f(U) \subset g(U)$.

Key words and phrases. Multivalent functions, differential subordination, differential superoordination, dominant, subordinant, differential operator.

2010 Mathematics Subject Classification. Primary: 30C45. Secondary: 30C80.

DOI 10.46793/KgJMat2101.007W

Received: May 16, 2018.

Accepted: August 22, 2018. 
Let $\xi, h \in H$ and $\psi(r, s, t ; z): \mathbb{C}^{3} \times U \rightarrow \mathbb{C}$. If $\xi$ and $\psi\left(\xi(z), z \xi^{\prime}(z), z^{2} \xi^{\prime \prime}(z) ; z\right)$ are univalent functions in $U$ and if $\xi$ satisfies the second-order differential superordination

$$
h(z) \prec \psi\left(\xi(z), z \xi^{\prime}(z), z^{2} \xi^{\prime \prime}(z) ; z\right),
$$

then $\xi$ is called a solution of the differential superordination (1.1). (If $f$ is subordinate to $g$, then $g$ is superordinate to $f$.) An analytic function $q$ is called a subordinant of (1.1), if $q \prec \xi$ for all $\xi$ satisfying (1.1). An univalent subordinant $\widetilde{q}$ that satisfies $q \prec \widetilde{q}$ for all the subordinants $q$ of (1.1) is called the best subordinant.

Recently, Miller and Mocanu [11] obtained conditions on the functions $h, q$ and $\psi$ for which the following implication holds:

$$
h(z) \prec \psi\left(\xi(z), z \xi^{\prime}(z), z^{2} \xi^{\prime \prime}(z) ; z\right) \Rightarrow q(z) \prec \xi(z) .
$$

Using the results due to Miller and Mocanu [11], Bulboacă [4] considered certain classes of first order differential superordination as well as superordination-preserving integral operators [5]. Ali et al. [1] have used the results of Bulboacă [4] to obtain sufficient conditions for certain normalized analytic functions to satisfy

$$
q_{1}(z) \prec \frac{z f^{\prime}(z)}{f(z)} \prec q_{2}(z)
$$

where $q_{1}$ and $q_{2}$ are given univalent functions in $U$ with $q_{1}(0)=q_{2}(0)=1$.

Very recently, Shanmugam et al. [17-19] and Goyal et al. [9] have obtained sandwich results for certain classes of analytic functions.

For $m, n \in \mathbb{N}_{0}=\mathbb{N} \cup\{0\}, \lambda_{1} \geq \lambda_{2} \geq 0$ and $f \in A_{p}$, the differential operator $D_{\lambda_{1}, \lambda_{2}, p}^{m, n}$ (see [8]) is defined by

$$
D_{\lambda_{1}, \lambda_{2}, p}^{m, n} f(z)=z^{p}+\sum_{k=p+1}^{\infty}\left[\frac{p+\left(\lambda_{1}+\lambda_{2}\right)(k-p)}{p+\lambda_{2}(k-p)}\right]^{m} C(k, n) a_{k} z^{k},
$$

where $C(k, n)=\frac{\Gamma(k+n)}{\Gamma(k)}$.

It follows from (1.2) that

$$
\begin{aligned}
\lambda_{1} z\left(D_{\lambda_{1}, \lambda_{2}, p}^{m, n} f(z)\right)^{\prime}= & \left(p+\lambda_{2}(k-p)\right) D_{\lambda_{1}, \lambda_{2}, p}^{m+1, n} f(z) \\
& -\left(p+\lambda_{2}(k-p)-p \lambda_{1}\right) D_{\lambda_{1}, \lambda_{2}, p}^{m, n} f(z), \quad \lambda_{1}>0 .
\end{aligned}
$$

Special cases of this operator includes the Ruscheweyh derivative operator [15], the Sălăgean derivative operator [16], the generalized Sălăgean operator [2], the generalized Ruscheweyh derivative operator [3], the generalized Al-Shaqsi and Darus derivative operator [6].

The main object of the present paper is to derive the several subordination and superordination results for multivalent analytic functions involving differential operator $D_{\lambda_{1}, \lambda_{2}, p}^{m, n}$.

In order to prove our results, we make use of the following known results. 
Definition $1.1([10])$. Denote by $Q$ the set of all functions $f$ that are analytic and injective on $\bar{U} \backslash E(f)$, where

$$
E(f)=\left\{\zeta \in \partial U: \lim _{z \rightarrow \zeta} f(z)=\infty\right\}
$$

and are such that $f^{\prime}(\zeta) \neq 0$ for $\zeta \in \partial U \backslash E(f)$.

Lemma 1.1 ([10]). Let $q$ be univalent in the unit disk $U$ and let $\theta$ and $\phi$ be analytic in a domain $D$ containing $q(U)$ with $\phi(w) \neq 0$ when $w \in q(U)$. Set $Q(z)=z q^{\prime}(z) \phi(q(z))$ and $h(z)=\theta(q(z))+Q(z)$. Suppose that

(1) $Q(z)$ is starlike univalent in $U$;

(2) $\operatorname{Re}\left(\frac{z h^{\prime}(z)}{Q(z)}\right)>0$ for $z \in U$.

If $\xi$ is analytic in $U$, with $\xi(0)=q(0), \xi(U) \subset D$ and

$$
\theta(\xi(z))+z \xi^{\prime}(z) \phi(\xi(z)) \prec \theta(q(z))+z q^{\prime}(z) \phi(q(z)),
$$

then $\xi \prec q$ and $q$ is the best dominant of (1.4).

Lemma 1.2 ([11]). Let $q$ be a convex univalent function in $U$ and let $\alpha \in \mathbb{C}, \beta \in$ $\mathbb{C} \backslash\{0\}$ with

$$
\operatorname{Re}\left(1+\frac{z q^{\prime \prime}(z)}{q^{\prime}(z)}\right)>\max \left\{0,-\operatorname{Re}\left(\frac{\alpha}{\beta}\right)\right\} .
$$

If $\xi$ is analytic in $U$ and

$$
\alpha \xi(z)+\beta z \xi^{\prime}(z) \leq \alpha q(z)+\beta z q^{\prime}(z),
$$

then $\xi \prec q$ and $q$ is the best dominant of (1.5).

Lemma $1.3([11])$. Let $q$ be convex univalent in $U$ and let $\beta \in \mathbb{C}$. Further assume that $\operatorname{Re}(\beta)>0$. If $\xi \in H[q(0), 1] \cap Q$ and $\xi(z)+\beta z \xi^{\prime}(z)$ is univalent in $U$, then

$$
q(z)+\beta z q^{\prime}(z) \prec \xi(z)+\beta z \xi^{\prime}(z)
$$

which implies that $q \prec \xi$ and $q$ is the best subordinant of (1.6).

Lemma $1.4([4])$. Let $q$ be convex univalent in the unit disk $U$ and let $\theta$ and $\phi$ be analytic in a domain $D$ containing $q(U)$. Suppose that

(1) $\operatorname{Re}\left(\frac{\theta^{\prime}(q(z))}{\phi(q(z))}\right)>0$ for $z \in U$;

(2) $Q(z)=z q^{\prime}(z \phi(q(z)))$ is starlike univalent in $U$.

If $\xi \in H[q(0), 1] \cap Q$, with $\xi(U) \subset D, \phi(\xi(z))+z \xi^{\prime}(z) \phi(\xi(z))$ is univalent in $U$ and

$$
\theta(q(z))+z q^{\prime}(z) \phi(q(z)) \prec \theta(\xi(z))+z \xi^{\prime}(z) \phi(\xi(z)),
$$

then $q \prec \xi$ and $q$ is the best subordinant of (1.7). 


\section{Main Results}

Theorem 2.1. Let $q$ be convex univalent in $U$ with $q(0)=1, \sigma \in \mathbb{C} \backslash\{0\}, \gamma>0$ and suppose that $q$ satisfies

$$
\operatorname{Re}\left(1+\frac{z q^{\prime \prime}(z)}{q^{\prime}(z)}\right)>\max \left\{0,-\operatorname{Re}\left(\frac{p \gamma}{\sigma}\right)\right\}
$$

If $f \in A_{p}$ satisfies the subordination

$$
\begin{aligned}
& \left(1-\frac{\sigma\left(p+\lambda_{2}(k-p)\right)}{\lambda_{1} p}\right)\left(\frac{D_{\lambda_{1}, \lambda_{2}, p}^{m, n} f(z)}{z^{p}}\right)^{\gamma} \\
& +\frac{\sigma\left(p+\lambda_{2}(k-p)\right)}{\lambda_{1} p}\left(\frac{D_{\lambda_{1}, \lambda_{2}, p}^{m, n} f(z)}{z^{p}}\right)^{\gamma}\left(\frac{D_{\lambda_{1}, \lambda_{2}, p}^{m+1, n} f(z)}{D_{\lambda_{1}, \lambda_{2}, p}^{m, n} f(z)}\right) \prec q(z)+\frac{\sigma}{p \gamma} z q^{\prime}(z),
\end{aligned}
$$

then

$$
\left(\frac{D_{\lambda_{1}, \lambda_{2}, p}^{m, n} f(z)}{z^{p}}\right)^{\gamma} \prec q(z)
$$

and $q$ is the best dominant of (2.2).

Proof. Define the function $\xi$ by

$$
\xi(z)=\left(\frac{D_{\lambda_{1}, \lambda_{2}, p}^{m, n} f(z)}{z^{p}}\right)^{\gamma}, \quad z \in U
$$

Differentiating (2.4) logarithmically with respect to $z$, we get

$$
\frac{z \xi^{\prime}(z)}{\xi(z)}=\gamma\left(\frac{z\left(D_{\lambda_{1}, \lambda_{2}, p}^{m, n} f(z)\right)^{\prime}}{D_{\lambda_{1}, \lambda_{2}, p}^{m, p} f(z)}-p\right)
$$

Now, in view of (1.3), we obtain the following subordination

$$
\frac{z \xi^{\prime}(z)}{\xi(z)}=\frac{\gamma\left(p+\lambda_{2}(k-p)\right)}{\lambda_{1}}\left(\frac{D_{\lambda_{1}, \lambda_{2}, p}^{m+\lambda_{1, n}} f(z)}{D_{\lambda_{1}, \lambda_{2}, p}^{m, n} f(z)}-1\right) .
$$

Therefore,

$$
\frac{z \xi^{\prime}(z)}{p \gamma}=\frac{\left(p+\lambda_{2}(k-p)\right)}{\lambda_{1} p}\left(\frac{D_{\lambda_{1}, \lambda_{2}, p}^{m, n} f(z)}{z^{p}}\right)^{\gamma}\left(\frac{D_{\lambda_{1}, \lambda_{2}, p}^{m+\lambda_{1}, n} f(z)}{D_{\lambda_{1}, \lambda_{2}, p}^{m, n} f(z)}-1\right) .
$$

The subordination (2.2) from the hypothesis becomes

$$
\xi(z)+\frac{\sigma}{p \gamma} z \xi^{\prime}(z) \prec q(z)+\frac{\sigma}{p \gamma} z q^{\prime}(z) .
$$

Hence, an application of Lemma 1.2 with $\alpha=1$ and $\beta=\frac{\sigma}{p \gamma}$, we obtain (2.3). 
Theorem 2.2. Let $\eta_{i} \in \mathbb{C}, i=1,2,3,4, \gamma>0, \delta \in \mathbb{C} \backslash\{0\}$ and $q$ be convex univalent in $U$ with $q(0)=1, q(z) \neq 0(z \in U)$ and assume that $q$ satisfies

$$
\operatorname{Re}\left(1+\frac{\eta_{2}}{\delta} q(z)+\frac{2 \eta_{3}}{\delta} q^{2}(z)+\frac{3 \eta_{4}}{\delta} q^{3}(z)+\frac{z q^{\prime \prime}(z)}{q^{\prime}(z)}-\frac{z q^{\prime}(z)}{q(z)}\right)>0 .
$$

Suppose that $\frac{z q^{\prime}(z)}{q(z)}$ is starlike univalent in $U$. If $f \in A_{p}$ satisfies

$$
\Omega_{1}\left(\eta_{i}\right)_{1}^{4}\left(\gamma, \delta, m, n, \lambda_{1}, \lambda_{2}, p ; z\right) \prec \eta_{1}+\eta_{2} q(z)+\eta_{3} q^{2}(z)+\eta_{4} q^{3}(z)+\delta \frac{z q^{\prime}(z)}{q(z)}
$$

where

$$
\begin{aligned}
& \Omega_{1}\left(\eta_{i}\right)_{1}^{4}\left(\gamma, \delta, m, n, \lambda_{1}, \lambda_{2}, p ; z\right)=\Omega_{1}\left(\eta_{1}, \eta_{2}, \eta_{3}, \eta_{4}, \gamma, \delta, m, n, \lambda_{1}, \lambda_{2}, p ; z\right) \\
= & \eta_{1}+\eta_{2}\left(\frac{D_{\lambda_{1}, \lambda_{2}, p}^{m+1, n} f(z)}{D_{\lambda_{1}, \lambda_{2}, p}^{m,} f(z)}\right)^{\gamma}+\eta_{3}\left(\frac{D_{\lambda_{1}, \lambda_{2}, p}^{m+1, n} f(z)}{D_{\lambda_{1}, \lambda_{2}, p}^{m, n} f(z)}\right)^{2 \gamma}+\eta_{4}\left(\frac{D_{\lambda_{1}, \lambda_{2}, p}^{m+1, n} f(z)}{D_{\lambda_{1}, \lambda_{2}, p}^{m, n} f(z)}\right)^{3 \gamma} \\
& +\frac{\gamma \delta\left(p+\lambda_{2}(k-p)\right)}{\lambda_{1}}\left(\frac{D_{\lambda_{1}, \lambda_{2}, p}^{m+2, n} f(z)}{D_{\lambda_{1}, \lambda_{2}, p}^{m+1, n} f(z)}-\frac{D_{\lambda_{1}, \lambda_{2}, p}^{m+1, n} f(z)}{D_{\lambda_{1}, \lambda_{2}, p}^{m, n} f(z)}\right)
\end{aligned}
$$

then

$$
\left(\frac{D_{\lambda_{1}, \lambda_{2}, p}^{m+1, n} f(z)}{D_{\lambda_{1}, \lambda_{2}, p}^{m, n} f(z)}\right)^{\gamma} \prec q(z)
$$

and $q$ is the best dominant of (2.6).

Proof. Define the function $\xi$ by

$$
\xi(z)=\left(\frac{D_{\lambda_{1}, \lambda_{2}, p}^{m+1, n} f(z)}{D_{\lambda_{1}, \lambda_{2}, p}^{m, n} f(z)}\right)^{\gamma}, \quad z \in U
$$

By a straightforward computation and using (1.3), we have

$$
\eta_{1}+\eta_{2} \xi(z)+\eta_{3} \xi^{2}(z)+\eta_{4} \xi^{3}(z)+\delta \frac{z \xi^{\prime}(z)}{\xi(z)}=\Omega_{1}\left(\eta_{i}\right)_{1}^{4}\left(\gamma, \delta, m, n, \lambda_{1}, \lambda_{2}, p ; z\right)
$$

where $\Omega_{1}\left(\eta_{i}\right)_{1}^{4}\left(\gamma, \delta, m, n, \lambda_{1}, \lambda_{2}, p ; z\right)$ is given by $(2.7)$.

From (2.6) and (2.9), we obtain

$\eta_{1}+\eta_{2} \xi(z)+\eta_{3} \xi^{2}(z)+\eta_{4} \xi^{3}(z)+\delta \frac{z \xi^{\prime}(z)}{\xi(z)} \prec \eta_{1}+\eta_{2} q(z)+\eta_{3} q^{2}(z)+\eta_{4} q^{3}(z)+\delta \frac{z q^{\prime}(z)}{q(z)}$.

By setting $\theta(w)=\eta_{1}+\eta_{2} w+\eta_{3} w^{2}+\eta_{4} w^{3}$ and $\phi(w)=\frac{\delta}{w}, w \neq 0$, we see that $\theta(w)$ is analytic in $\mathbb{C}, \phi(w)$ is analytic in $\mathbb{C} \backslash\{0\}$ and that $\phi(w) \neq 0, w \in \mathbb{C} \backslash\{0\}$. Also, we get

$$
Q(z)=z q^{\prime}(z) \phi(q(z))=\delta \frac{z q^{\prime}(z)}{q(z)}
$$


and

$$
h(z)=\theta(q(z))+Q(z)=\eta_{1}+\eta_{2} q(z)+\eta_{3} q^{2}(z)+\eta_{4} q^{3}(z)+\delta \frac{z q^{\prime}(z)}{q(z)} .
$$

It is clear that $Q(z)$ is starlike univalent in $U$,

$$
\operatorname{Re}\left(\frac{z h^{\prime}(z)}{Q(z)}\right)=\operatorname{Re}\left(1+\frac{\eta_{2}}{\delta} q(z)+\frac{2 \eta_{3}}{\delta} q^{2}(z)+\frac{3 \eta_{4}}{\delta} q^{3}(z)+\frac{z q^{\prime \prime}(z)}{q^{\prime}(z)}-\frac{z q^{\prime}(z)}{q(z)}\right)>0 .
$$

Thus, by Lemma 1.1, we get $\xi(z) \prec q(z)$. By using (2.8), we obtain the desired result.

Theorem 2.3. Let $\eta_{i} \in \mathbb{C}, i=1,2,3,4, \gamma>0, \delta \in \mathbb{C} \backslash\{0\}$ and $q$ be convex univalent in $U$ with $q(0)=1, q(z) \neq 0, z \in U$, and assume that $q$ satisfies (2.5). Suppose that $\frac{z q^{\prime}(z)}{q(z)}$ is starlike univalent in $U$. If $f \in A_{p}$ satisfies

$$
\Omega_{2}\left(\eta_{i}\right)_{1}^{4}\left(\gamma, \delta, m, n, \lambda_{1}, \lambda_{2}, p ; z\right) \prec \eta_{1}+\eta_{2} q(z)+\eta_{3} q^{2}(z)+\eta_{4} q^{3}(z)+\delta \frac{z q^{\prime}(z)}{q(z)}
$$

where

$$
\begin{aligned}
& \Omega_{2}\left(\eta_{i}\right)_{1}^{4}\left(\gamma, \delta, m, n, \lambda_{1}, \lambda_{2}, p ; z\right)=\Omega_{2}\left(\eta_{1}, \eta_{2}, \eta_{3}, \eta_{4}, \gamma, \delta, m, n, \lambda_{1}, \lambda_{2}, p ; z\right) \\
= & \eta_{1}+\eta_{2}\left(\frac{D_{\lambda_{1}, \lambda_{2}, p}^{m, n} f(z)}{D_{\lambda_{1}, \lambda_{2}, p}^{m+1, n} f(z)}\right)^{\gamma}+\eta_{3}\left(\frac{D_{\lambda_{1}, \lambda_{2}, p}^{m} f(z)}{D_{\lambda_{1}, \lambda_{2}, p}^{m+1, n} f(z)}\right)^{2 \gamma}+\eta_{4}\left(\frac{D_{\lambda_{1}, \lambda_{2}, p}^{m, n} f(z)}{D_{\lambda_{1}, \lambda_{2}, p}^{m+1, n} f(z)}\right)^{3 \gamma} \\
& +\frac{\gamma \delta\left(p+\lambda_{2}(k-p)\right)}{\lambda_{1}}\left(\frac{D_{\lambda_{1}, \lambda_{2}, p}^{m+1, n} f(z)}{D_{\lambda_{1}, \lambda_{2}, p}^{m, n} f(z)}-\frac{D_{\lambda_{1}, \lambda_{2}, p}^{m+2, n} f(z)}{D_{\lambda_{1}, \lambda_{2}, p}^{m+1, n} f(z)}\right)
\end{aligned}
$$

then

$$
\left(\frac{D_{\lambda_{1}, \lambda_{2}, p}^{m, n} f(z)}{D_{\lambda_{1}, \lambda_{2}, p}^{m+1, n} f(z)}\right)^{\gamma} \prec q(z)
$$

and $q$ is the best dominant of (2.10).

Proof. The proof is similar to that of Theorem 2.2.

Theorem 2.4. Let $\eta_{i} \in \mathbb{C}, i=1,2,3,4, \delta \in \mathbb{C} \backslash\{0\}$ and $q$ be convex univalent in $U$ with $q(0)=1, q(z) \neq 0, z \in U$, and assume that $q$ satisfies $(2.5)$. Suppose that $\frac{z q^{\prime}(z)}{q(z)}$ is starlike univalent in $U$. If $f \in A_{p}$ satisfies

$$
\Omega_{3}\left(\eta_{i}\right)_{1}^{4}\left(\delta, m, n, \lambda_{1}, \lambda_{2}, p ; z\right) \prec \eta_{1}+\eta_{2} q(z)+\eta_{3} q^{2}(z)+\eta_{4} q^{3}(z)+\delta \frac{z q^{\prime}(z)}{q(z)}
$$


where

$$
\begin{aligned}
& \Omega_{3}\left(\eta_{i}\right)_{1}^{4}\left(\delta, m, n, \lambda_{1}, \lambda_{2}, p ; z\right)=\Omega_{3}\left(\eta_{1}, \eta_{2}, \eta_{3}, \eta_{4}, \gamma, \delta, m, n, \lambda_{1}, \lambda_{2}, p ; z\right) \\
= & \eta_{1}+\eta_{2} \frac{\left(D_{\lambda_{1}, \lambda_{2}, p}^{m, n} f(z)\right)^{2}}{z^{p} D_{\lambda_{1}, \lambda_{2}, p}^{m+1, n} f(z)}+\eta_{3} \frac{\left(D_{\lambda_{1}, \lambda_{2}, p}^{m, n} f(z)\right)^{4}}{z^{2 p}\left(D_{\lambda_{1}, \lambda_{2}, p}^{m+1, n} f(z)\right)^{2}}+\eta_{4} \frac{\left(D_{\lambda_{1}, \lambda_{2}, p}^{m, n} f(z)\right)^{6}}{z^{3 p}\left(D_{\lambda_{1}, \lambda_{2}, p}^{m+1, n} f(z)\right)^{3}} \\
& +\frac{\delta\left(p+\lambda_{2}(k-p)\right)}{\lambda_{1}}\left(\frac{2 D_{\lambda_{1}, \lambda_{2}, p}^{m+1, n} f(z)}{D_{\lambda_{1}, \lambda_{2}, p}^{m, n} f(z)}-\frac{D_{\lambda_{1}, \lambda_{2}, p}^{m+2, n} f(z)}{D_{\lambda_{1}, \lambda_{2}, p}^{m+1, n} f(z)}-1\right)
\end{aligned}
$$

then

$$
\frac{\left(D_{\lambda_{1}, \lambda_{2}, p}^{m, n} f(z)\right)^{2}}{z^{p} D_{\lambda_{1}, \lambda_{2}, p}^{m+1, n} f(z)} \prec q(z)
$$

and $q$ is the best dominant of (2.12).

Proof. Define the function $\xi$ by

$$
\xi(z)=\frac{\left(D_{\lambda_{1}, \lambda_{2}, p}^{m, n} f(z)\right)^{2}}{z^{p} D_{\lambda_{1}, \lambda_{2}, p}^{m+1, n} f(z)}, \quad z \in U .
$$

By a straightforward computation and using (1.3), we have

$$
\eta_{1}+\eta_{2} \xi(z)+\eta_{3} \xi^{2}(z)+\eta_{4} \xi^{3}(z)+\delta \frac{z \xi^{\prime}(z)}{\xi(z)}=\Omega_{3}\left(\eta_{i}\right)_{1}^{4}\left(\delta, m, n, \lambda_{1}, \lambda_{2}, p ; z\right),
$$

where $\Omega_{3}\left(\eta_{i}\right)_{1}^{4}\left(\delta, m, n, \lambda_{1}, \lambda_{2}, p ; z\right)$ is given by (2.13). From (2.12) and (2.15), we obtain

$$
\eta_{1}+\eta_{2} \xi(z)+\eta_{3} \xi^{2}(z)+\eta_{4} \xi^{3}(z)+\delta \frac{z \xi^{\prime}(z)}{\xi(z)} \prec \eta_{1}+\eta_{2} q(z)+\eta_{3} q^{2}(z)+\eta_{4} q^{3}(z)+\delta \frac{z q^{\prime}(z)}{q(z)} .
$$

The remaining part of Theorem 2.4 is similar to that of Theorem 2.2 and hence we omit it.

Theorem 2.5. Let $q$ be convex univalent in $U$ with $q(0)=1, \gamma>0$ and $\operatorname{Re}(\sigma)>0$. Let $f \in A_{p}$ satisfying

$$
\left(\frac{D_{\lambda_{1}, \lambda_{2}, p}^{m, n} f(z)}{z^{p}}\right)^{\gamma} \in H[q(0), 1] \cap Q
$$

and

$$
\begin{aligned}
& \left(1-\frac{\sigma\left(p+\lambda_{2}(k-p)\right)}{\lambda_{1} p}\right)\left(\frac{D_{\lambda_{1}, \lambda_{2}, p}^{m, n} f(z)}{z^{p}}\right)^{\gamma} \\
& +\frac{\sigma\left(p+\lambda_{2}(k-p)\right)}{\lambda_{1} p}\left(\frac{D_{\lambda_{1}, \lambda_{2}, p}^{m, n} f(z)}{z^{p}}\right)^{\gamma}\left(\frac{D_{\lambda_{1}, \lambda_{2}, p}^{m+1, n} f(z)}{D_{\lambda_{1}, \lambda_{2}, p}^{m, n} f(z)}\right)
\end{aligned}
$$


be univalent in $U$. If

$$
\begin{aligned}
q(z)+\frac{\sigma}{p \gamma} z q^{\prime}(z) \prec & \left(1-\frac{\sigma\left(p+\lambda_{2}(k-p)\right)}{\lambda_{1} p}\right)\left(\frac{D_{\lambda_{1}, \lambda_{2}, p}^{m, n} f(z)}{z^{p}}\right)^{\gamma} \\
& +\frac{\sigma\left(p+\lambda_{2}(k-p)\right)}{\lambda_{1} p}\left(\frac{D_{\lambda_{1}, \lambda_{2}, p}^{m, n} f(z)}{z^{p}}\right)^{\gamma}\left(\frac{D_{\lambda_{1}, \lambda_{2}, p}^{m+1, n} f(z)}{D_{\lambda_{1}, \lambda_{2}, p}^{m, n} f(z)}\right)
\end{aligned}
$$

then

$$
q(z) \prec\left(\frac{D_{\lambda_{1}, \lambda_{2}, p}^{m, n} f(z)}{z^{p}}\right)^{\gamma}
$$

and $q$ is the best subordinant of (2.16).

Proof. Define the function $\xi$ by

$$
\xi(z)=\left(\frac{D_{\lambda_{1}, \lambda_{2}, p}^{m, n} f(z)}{z^{p}}\right)^{\gamma} .
$$

Differentiating (2.18) logarithmically with respect to $z$, we get

$$
\frac{z \xi^{\prime}(z)}{\xi(z)}=\gamma\left(\frac{z\left(D_{\lambda_{1}, \lambda_{2}, p}^{m, n} f(z)\right)^{\prime}}{D_{\lambda_{1}, \lambda_{2}, p}^{m, n} f(z)}-p\right) .
$$

After some computations and using (1.3), from (2.19), we have

$$
\begin{aligned}
& \left(1-\frac{\sigma\left(p+\lambda_{2}(k-p)\right)}{\lambda_{1} p}\right)\left(\frac{D_{\lambda_{1}, \lambda_{2}, p}^{m, n} f(z)}{z^{p}}\right)^{\gamma} \\
& +\frac{\sigma\left(p+\lambda_{2}(k-p)\right)}{\lambda_{1} p}\left(\frac{D_{\lambda_{1}, \lambda_{2}, p}^{m, n} f(z)}{z^{p}}\right)^{\gamma}\left(\frac{D_{\lambda_{1}, \lambda_{2}, p}^{m+1, n} f(z)}{D_{\lambda_{1}, \lambda_{2}, p}^{m, n} f(z)}\right)=\xi(z)+\frac{\sigma}{p \gamma} z \xi^{\prime}(z) .
\end{aligned}
$$

From (2.16) and (2.20), we get

$$
q(z)+\frac{\sigma}{p \gamma} z q^{\prime}(z) \prec \xi(z)+\frac{\sigma}{p \gamma} z \xi^{\prime}(z) .
$$

Hence, an application of Lemma 1.3 with $\alpha=1$ and $\beta=\frac{\sigma}{p \gamma}$, we obtain (2.17).

Theorem 2.6. Let $\eta_{i} \in \mathbb{C}, i=1,2,3,4, \gamma>0, \delta \in \mathbb{C} \backslash\{0\}$ and $q$ be convex univalent in $U$ with $q(0)=1, q(z) \neq 0, z \in U$ and assume that $q$ satisfies

$$
\operatorname{Re}\left(\frac{\eta_{2}}{\delta} q(z)+\frac{2 \eta_{3}}{\delta} q^{2}(z)+\frac{3 \eta_{4}}{\delta} q^{3}(z)\right)>0 .
$$

Suppose that $\frac{z q^{\prime}(z)}{q(z)}$ is starlike univalent in $U$. Let $f \in A_{p}$ satisfying

$$
\left(\frac{D_{\lambda_{1}, \lambda_{2}, p}^{m+1, n} f(z)}{D_{\lambda_{1}, \lambda_{2}, p}^{m, n} f(z)}\right)^{\gamma} \in H[q(0), 1] \cap Q
$$


and $\Omega_{1}\left(\eta_{i}\right)_{1}^{4}\left(\gamma, \delta, m, n, \lambda_{1}, \lambda_{2}, p ; z\right)$ be univalent in $U$, where $\Omega_{1}\left(\eta_{i}\right)_{1}^{4}\left(\gamma, \delta, m, n, \lambda_{1}, \lambda_{2}, p ; z\right)$ is given by $(2.7)$. If

$(2.22) \eta_{1}+\eta_{2} q(z)+\eta_{3} q^{2}(z)+\eta_{4} q^{3}(z)+\delta \frac{z q^{\prime}(z)}{q(z)} \prec \Omega_{1}\left(\eta_{i}\right)_{1}^{4}\left(\gamma, \delta, m, n, \lambda_{1}, \lambda_{2}, p ; z\right)$,

then

$$
q(z) \prec\left(\frac{D_{\lambda_{1}, \lambda_{2}, p}^{m+1, n} f(z)}{D_{\lambda_{1}, \lambda_{2}, p}^{m, n} f(z)}\right)^{\gamma}
$$

and $q$ is the best subordinant of (2.22).

Proof. Define the function $\xi$ by

$$
\xi(z)=\left(\frac{D_{\lambda_{1}, \lambda_{2}, p}^{m+1, n} f(z)}{D_{\lambda_{1}, \lambda_{2}, p}^{m, n} f(z)}\right)^{\gamma}, \quad z \in U
$$

By a straightforward computation, we have

$$
\Omega_{1}\left(\eta_{i}\right)_{1}^{4}\left(\gamma, \delta, m, n, \lambda_{1}, \lambda_{2}, p ; z\right)=\eta_{1}+\eta_{2} \xi(z)+\eta_{3} \xi^{2}(z)+\eta_{4} \xi^{3}(z)+\delta \frac{z \xi^{\prime}(z)}{\xi(z)}
$$

where $\Omega_{1}\left(\eta_{i}\right)_{1}^{4}\left(\gamma, \delta, m, n, \lambda_{1}, \lambda_{2}, p ; z\right)$ is given by $(2.7)$.

From (2.22) and (2.24), we obtain

$$
\begin{gathered}
\eta_{1}+\eta_{2} q(z)+\eta_{3} q^{2}(z)+\eta_{4} q^{3}(z)+\delta \frac{z q^{\prime}(z)}{q(z)} \\
\prec \eta_{1}+\eta_{2} \xi(z)+\eta_{3} \xi^{2}(z)+\eta_{4} \xi^{3}(z)+\delta \frac{z \xi^{\prime}(z)}{\xi(z)} .
\end{gathered}
$$

By setting $\theta(w)=\eta_{1}+\eta_{2} w+\eta_{3} w^{2}+\eta_{4} w^{3}$ and $\phi(w)=\frac{\delta}{w}, w \neq 0$, we see that $\theta(w)$ is analytic in $\mathbb{C}, \phi(w)$ is analytic in $\mathbb{C} \backslash\{0\}$ and that $\phi(w) \neq 0, w \in \mathbb{C} \backslash\{0\}$. Also, we get

$$
Q(z)=z q^{\prime}(z) \phi(q(z))=\delta \frac{z q^{\prime}(z)}{q(z)} .
$$

It is clear that $Q(z)$ is starlike univalent in $U$,

$$
\operatorname{Re}\left(\frac{\theta^{\prime}(q(z))}{\phi(q(z))}\right)=\operatorname{Re}\left(\frac{\eta_{2}}{\delta} q(z)+\frac{2 \eta_{3}}{\delta} q^{2}(z)+\frac{3 \eta_{4}}{\delta} q^{3}(z)\right)>0 .
$$

Thus, by Lemma 1.4, we get $q(z) \prec \xi(z)$. By using (2.23), we obtain the desired result.

Theorem 2.7. Let $\eta_{i} \in \mathbb{C}, i=1,2,3,4, \gamma>0, \delta \in \mathbb{C} \backslash\{0\}$ and $q$ be convex univalent in $U$ with $q(0)=1, q(z) \neq 0(z \in U)$ and assume that $q$ satisfies (2.21). Suppose that $\frac{z q^{\prime}(z)}{q(z)}$ is starlike univalent in $U$. Let $f \in A_{p}$ satisfying

$$
\left(\frac{D_{\lambda_{1}, \lambda_{2}, p}^{m, n} f(z)}{D_{\lambda_{1}, \lambda_{2}, p}^{m+1, n} f(z)}\right)^{\gamma} \in H[q(0), 1] \cap Q
$$


and $\Omega_{2}\left(\eta_{i}\right)_{1}^{4}\left(\gamma, \delta, m, n, \lambda_{1}, \lambda_{2}, p ; z\right)$ be univalent in $U$, where $\Omega_{2}\left(\eta_{i}\right)_{1}^{4}\left(\gamma, \delta, m, n, \lambda_{1}, \lambda_{2}, p ; z\right)$ is given by $(2.11)$. If

$$
\eta_{1}+\eta_{2} q(z)+\eta_{3} q^{2}(z)+\eta_{4} q^{3}(z)+\delta \frac{z q^{\prime}(z)}{q(z)} \prec \Omega_{2}\left(\eta_{i}\right)_{1}^{4}\left(\gamma, \delta, m, n, \lambda_{1}, \lambda_{2}, p ; z\right),
$$

then

$$
q(z) \prec\left(\frac{D_{\lambda_{1}, \lambda_{2}, p}^{m, n} f(z)}{D_{\lambda_{1}, \lambda_{2}, p}^{m+1, n} f(z)}\right)^{\gamma}
$$

and $q$ is the best subordinant of (2.25).

Proof. The proof is similar to that of Theorem 2.6.

Theorem 2.8. Let $\eta_{i} \in \mathbb{C}, i=1,2,3,4, \delta \in \mathbb{C} \backslash\{0\}$ and $q$ be convex univalent in $U$ with $q(0)=1, q(z) \neq 0, z \in U$, and assume that $q$ satisfies (2.21). Suppose that $\frac{z q^{\prime}(z)}{q(z)}$ is starlike univalent in $U$. Let $f \in A_{p}$ satisfying

$$
\frac{\left(D_{\lambda_{1}, \lambda_{2}, p}^{m, n} f(z)\right)^{2}}{z^{p} D_{\lambda_{1}, \lambda_{2}, p}^{m+1, n} f(z)} \in H[q(0), 1] \cap Q
$$

and $\Omega_{3}\left(\eta_{i}\right)_{1}^{4}\left(\delta, m, n, \lambda_{1}, \lambda_{2}, p ; z\right)$ be univalent in $U$, where $\Omega_{3}\left(\eta_{i}\right)_{1}^{4}\left(\delta, m, n, \lambda_{1}, \lambda_{2}, p ; z\right)$ is given by (2.13). If

$$
\eta_{1}+\eta_{2} q(z)+\eta_{3} q^{2}(z)+\eta_{4} q^{3}(z)+\delta \frac{z q^{\prime}(z)}{q(z)} \prec \Omega_{3}\left(\eta_{i}\right)_{1}^{4}\left(\delta, m, n, \lambda_{1}, \lambda_{2}, p ; z\right),
$$

then

$$
q(z) \prec \frac{\left(D_{\lambda_{1}, \lambda_{2}, p}^{m, n} f(z)\right)^{2}}{z^{p} D_{\lambda_{1}, \lambda_{2}, p}^{m+1, n} f(z)}
$$

and $q$ is the best subordinant of (2.26).

Proof. Define the function $\xi$ by

$$
\xi(z)=\frac{\left(D_{\lambda_{1}, \lambda_{2}, p}^{m, n} f(z)\right)^{2}}{z^{p} D_{\lambda_{1}, \lambda_{2}, p}^{m+1, n} f(z)}, \quad z \in U .
$$

By a straightforward computation and using (1.3), we have

$$
\Omega_{3}\left(\eta_{i}\right)_{1}^{4}\left(\delta, m, n, \lambda_{1}, \lambda_{2}, p ; z\right)=\eta_{1}+\eta_{2} \xi(z)+\eta_{3} \xi^{2}(z)+\eta_{4} \xi^{3}(z)+\delta \frac{z \xi^{\prime}(z)}{\xi(z)},
$$

where $\Omega_{3}\left(\eta_{i}\right)_{1}^{4}\left(\delta, m, n, \lambda_{1}, \lambda_{2}, p ; z\right)$ is given by $(2.13)$.

From (2.26) and (2.27), we obtain

$$
\begin{aligned}
& \eta_{1}+\eta_{2} q(z)+\eta_{3} q^{2}(z)+\eta_{4} q^{3}(z)+\delta \frac{z q^{\prime}(z)}{q(z)} \\
& \prec \eta_{1}+\eta_{2} \xi(z)+\eta_{3} \xi^{2}(z)+\eta_{4} \xi^{3}(z)+\delta \frac{z \xi^{\prime}(z)}{\xi(z)} .
\end{aligned}
$$


The remaining part of Theorem 2.8 is similar to that of Theorem 2.6 and hence we omit it.

Concluding the results of differential subordination and superordination, we state the following "sandwich results".

Theorem 2.9. Let $q_{1}$ and $q_{2}$ be convex univalent in $U$ with $q_{1}(0)=q_{2}(0)=1$. Suppose $q_{2}$ satisfies (2.1), $\gamma>0$ and $\operatorname{Re}(\sigma)>0$. Let $f \in A_{p}$ satisfying

$$
\left(\frac{D_{\lambda_{1}, \lambda_{2}, p}^{m, n} f(z)}{z^{p}}\right)^{\gamma} \in H[1,1] \cap Q
$$

and

$$
\begin{aligned}
& \left(1-\frac{\sigma\left(p+\lambda_{2}(k-p)\right)}{\lambda_{1} p}\right)\left(\frac{D_{\lambda_{1}, \lambda_{2}, p}^{m, n} f(z)}{z^{p}}\right)^{\gamma} \\
& +\frac{\sigma\left(p+\lambda_{2}(k-p)\right)}{\lambda_{1} p}\left(\frac{D_{\lambda_{1}, \lambda_{2}, p}^{m, n} f(z)}{z^{p}}\right)^{\gamma}\left(\frac{D_{\lambda_{1}, \lambda_{2}, p}^{m+1, n} f(z)}{D_{\lambda_{1}, \lambda_{2}, p}^{m, n} f(z)}\right),
\end{aligned}
$$

be univalent in $U$. If

$$
\begin{aligned}
& q_{1}(z)+\frac{\sigma}{p \gamma} z q_{1}^{\prime}(z) \prec\left(1-\frac{\sigma\left(p+\lambda_{2}(k-p)\right)}{\lambda_{1} p}\right)\left(\frac{D_{\lambda_{1}, \lambda_{2}, p}^{m, n} f(z)}{z^{p}}\right)^{\gamma} \\
& +\frac{\sigma\left(p+\lambda_{2}(k-p)\right)}{\lambda_{1} p}\left(\frac{D_{\lambda_{1}, \lambda_{2}, p}^{m, n} f(z)}{z^{p}}\right)^{\gamma}\left(\frac{D_{\lambda_{1}, \lambda_{2}, p}^{m+1, n} f(z)}{D_{\lambda_{1}, \lambda_{2}, p}^{m, n} f(z)}\right) \prec q_{2}(z)+\frac{\sigma}{p \gamma} z q_{2}^{\prime}(z),
\end{aligned}
$$

then

$$
q_{1}(z) \prec\left(\frac{D_{\lambda_{1}, \lambda_{2}, p}^{m, n} f(z)}{z^{p}}\right)^{\gamma} \prec q_{2}(z)
$$

and $q_{1}$ and $q_{2}$ are, respectively, the best subordinant and the best dominant.

Theorem 2.10. Let $q_{1}$ and $q_{2}$ be convex univalent in $U$ with $q_{1}(0)=q_{2}(0)=1$. Suppose $q_{1}$ satisfies (2.21) and $q_{2}$ satisfies (2.5). Let $f \in A_{p}$ satisfying

$$
\left(\frac{D_{\lambda_{1}, \lambda_{2}, p}^{m+1, n} f(z)}{D_{\lambda_{1}, \lambda_{2}, p}^{m, n} f(z)}\right)^{\gamma} \in H[1,1] \cap Q
$$

and $\Omega_{1}\left(\eta_{i}\right)_{1}^{4}\left(\gamma, \delta, m, n, \lambda_{1}, \lambda_{2}, p ; z\right)$ be univalent in $U$, where $\Omega_{1}\left(\eta_{i}\right)_{1}^{4}\left(\gamma, \delta, m, n, \lambda_{1}, \lambda_{2}, p ; z\right)$ is given by $(2.7)$. If

$$
\begin{aligned}
\eta_{1}+\eta_{2} q_{1}(z)+\eta_{3} q_{1}^{2}(z)+\eta_{4} q_{1}^{3}(z)+\delta \frac{z q_{1}^{\prime}(z)}{q_{1}(z)} & \prec \Omega_{1}\left(\eta_{i}\right)_{1}^{4}\left(\gamma, \delta, m, n, \lambda_{1}, \lambda_{2}, p ; z\right) \\
\prec & \eta_{1}+\eta_{2} q_{2}(z)+\eta_{3} q_{2}^{2}(z)+\eta_{4} q_{2}^{3}(z) \\
& +\delta \frac{z q_{2}^{\prime}(z)}{q_{2}(z)}
\end{aligned}
$$


then

$$
q_{1}(z) \prec\left(\frac{D_{\lambda_{1}, \lambda_{2}, p}^{m+1, n} f(z)}{D_{\lambda_{1}, \lambda_{2}, p}^{m, n} f(z)}\right)^{\gamma} \prec q_{2}(z)
$$

and $q_{1}$ and $q_{2}$ are, respectively, the best subordinant and the best dominant.

Theorem 2.11. Let $q_{1}$ and $q_{2}$ be convex univalent in $U$ with $q_{1}(0)=q_{2}(0)=1$. Suppose $q_{1}$ satisfies (2.21) and $q_{2}$ satisfies (2.5). Let $f \in A_{p}$ satisfying

$$
\left(\frac{D_{\lambda_{1}, \lambda_{2}, p}^{m, n} f(z)}{D_{\lambda_{1}, \lambda_{2}, p}^{m+1, n} f(z)}\right)^{\gamma} \in H[1,1] \cap Q
$$

and $\Omega_{2}\left(\eta_{i}\right)_{1}^{4}\left(\gamma, \delta, m, n, \lambda_{1}, \lambda_{2}, p ; z\right)$ be univalent in $U$, where $\Omega_{2}\left(\eta_{i}\right)_{1}^{4}\left(\gamma, \delta, m, n, \lambda_{1}, \lambda_{2}, p ; z\right)$ is given by $(2.11)$. If

$$
\begin{aligned}
\eta_{1}+\eta_{2} q_{1}(z)+\eta_{3} q_{1}^{2}(z)+\eta_{4} q_{1}^{3}(z)+\delta \frac{z q_{1}^{\prime}(z)}{q_{1}(z)} & \prec \Omega_{2}\left(\eta_{i}\right)_{1}^{4}\left(\gamma, \delta, m, n, \lambda_{1}, \lambda_{2}, p ; z\right) \\
& \prec \eta_{1}+\eta_{2} q_{2}(z)+\eta_{3} q_{2}^{2}(z)+\eta_{4} q_{2}^{3}(z) \\
& +\delta \frac{z q_{2}^{\prime}(z)}{q_{2}(z)}
\end{aligned}
$$

then

$$
q_{1}(z) \prec\left(\frac{D_{\lambda_{1}, \lambda_{2}, p}^{m, n} f(z)}{D_{\lambda_{1}, \lambda_{2}, p}^{m+1, n} f(z)}\right)^{\gamma} \prec q_{2}(z)
$$

and $q_{1}$ and $q_{2}$ are, respectively, the best subordinant and the best dominant.

Theorem 2.12. Let $q_{1}$ and $q_{2}$ be convex univalent in $U$ with $q_{1}(0)=q_{2}(0)=1$. Suppose $q_{1}$ satisfies (2.21) and $q_{2}$ satisfies (2.5). Let $f \in A_{p}$ satisfying

$$
\frac{\left(D_{\lambda_{1}, \lambda_{2}, p}^{m, n} f(z)\right)^{2}}{z^{p} D_{\lambda_{1}, \lambda_{2}, p}^{m+1, n} f(z)} \in H[1,1] \cap Q
$$

and $\Omega_{3}\left(\eta_{i}\right)_{1}^{4}\left(\delta, m, n, \lambda_{1}, \lambda_{2}, p ; z\right)$ be univalent in $U$, where $\Omega_{3}\left(\eta_{i}\right)_{1}^{4}\left(\delta, m, n, \lambda_{1}, \lambda_{2}, p ; z\right)$ is given by $(2.13)$. If

$$
\begin{aligned}
\eta_{1}+\eta_{2} q_{1}(z) & +\eta_{3} q_{1}^{2}(z)+\eta_{4} q_{1}^{3}(z)+\delta \frac{z q_{1}^{\prime}(z)}{q_{1}(z)} \prec \Omega_{3}\left(\eta_{i}\right)_{1}^{4}\left(\delta, m, n, \lambda_{1}, \lambda_{2}, p ; z\right) \\
& \prec \eta_{1}+\eta_{2} q_{2}(z)+\eta_{3} q_{2}^{2}(z)+\eta_{4} q_{2}^{3}(z)+\delta \frac{z q_{2}^{\prime}(z)}{q_{2}(z)},
\end{aligned}
$$

then

$$
q_{1}(z) \prec \frac{\left(D_{\lambda_{1}, \lambda_{2}, p}^{m, n} f(z)\right)^{2}}{z^{p} D_{\lambda_{1}, \lambda_{2}, p}^{m+1, n} f(z)} \prec q_{2}(z)
$$

and $q_{1}$ and $q_{2}$ are, respectively, the best subordinant and the best dominant. 
Remark 2.1. By specifying the function $\phi$ and selecting the particular values of $\eta_{1}, \eta_{2}, \eta_{3}, \eta_{4}, \gamma, m, n, \lambda_{1}, \lambda_{2}$ and $p$, we can derive a number of known results. Some of them are given below.

(1) Taking $n=\lambda_{2}=0$ and $p=1$ in Theorems 2.1, 2.5, 2.9, we get the results obtained by Răducanu and Nechita [14, Theorem 3.1, Theorem 3.6, Theorem 3.9].

(2) Taking $n=\lambda_{2}=0$ and $\lambda_{1}=p=1$ in Theorems 2.1, 2.5, 2.9, we get the results obtained by Răducanu and Nechita [14, Corollary 3.3, Corollary 3.8, Corollary 3.11].

(3) Putting $n=m=\lambda_{2}=0$ and $\lambda_{1}=p=1$ in Theorem 2.1, we obtain the results obtained by Murugusundaramoorthy and Magesh [12, Corollary 3.3].

(4) Taking $n=m=\lambda_{2}=0$ and $\lambda_{1}=p=1$ in Theorems 2.5, 2.9, we obtain the results obtained by Răducanu and Nechita [14, Corollary 3.7, Corollary 3.10].

(5) For $\lambda_{2}=\eta_{1}=\eta_{2}=\eta_{4}=0, \gamma=p=1$ and $\phi(w)=\delta$ in Theorems 2.2, 2.6, 2.10, we have the results obtained by Darus and Al-Shaqsi [7, Theorem 2.1, Theorem 3.1, Theorem 3.3].

(6) By taking $n=\lambda_{2}=\eta_{1}=\eta_{3}=\eta_{4}=0, \gamma=\eta_{2}=p=1$ and $\phi(w)=\delta$ in Theorems 2.3, 2.7, 2.11, we get the results obtained by Nechita [13, Theorem 5, Theorem 10, Corollary 13].

(7) Putting $n=\lambda_{2}=\eta_{1}=\eta_{3}=\eta_{4}=0, \gamma=\lambda_{1}=\eta_{2}=p=1$ and $\phi(w)=\delta$ in Theorems 2.3, 2.7, 2.11, we obtain the results obtained by Shanmugan et al. [17, Theorem 5.1, Theorem 5.2, Theorem 5.3].

(8) Putting $n=m=\lambda_{2}=\eta_{1}=\eta_{3}=\eta_{4}=0, \gamma=\lambda_{1}=\eta_{2}=p=1$ and $\phi(w)=\delta$ in Theorems 2.3, 2.7, 2.11, we get the results obtained by Shanmugam et al. [17, Theorem 3.1, Theorem 3.2, Theorem 3.3].

\section{REFERENCES}

[1] R. M. Ali, V. Ravichandran, M. H. Khan and K. G. Subramanian, Differential sandwich theorems for certain analytic functions, Far East J. Math. Sci. 15(1) (2004), 87-94.

[2] F. M. Al-Oboudi, On univalent functions defined by a generalized Salagean operator, Int. J. Math. Math. Sci. 27 (2004), 1429-1436.

[3] K. Al-Shaqsi and M. Darus, On univalent functions with respect to $K$-symmetric points defined by a generalization Ruscheweyh derivative operators, J. Anal. Appl. 7 (2009), 53-61.

[4] T. Bulboacă, Classes of first order differential superordinations, Demonstr. Math. 35(2) (2002), 287-292.

[5] T. Bulboacă, A class of superordination-preserving integral operators, Indag. Math. (N.S.) 13(3) (2002), 301-311.

[6] M. Darus and K. Al-Shaqsi, Differential sandwich theorems with generalized derivative operator, Int. J. Comput. Math. Sci. 22 (2008), 75-78.

[7] M. Darus and K. Al-Shaqsi, Differential sandwich theorems with generalized derivative operator, Int. J. Math. Comput. Sci. 38 (2008), 11-14.

[8] E. A. Eljamal and M. Darus, Majorization for certain classes of analytic functions defined by a new operator, CUBO 14(1) (2012), 119-125. 
[9] S. P. Goyal, P. Goswami and H. Silverman, Subordination and superordination results for a class of analytic multivalent functions, Int. J. Math. Math. Sci. (2008), Article ID 561638, 1-12.

[10] S. S. Miller and P. T. Mocanu, Differential Subordinations: Theory and Applications, Series on Monographs and Textbooks in Pure and Applied Mathematics 225, Marcel Dekker Inc., New York, Basel, 2000.

[11] S. S. Miller and P. T. Mocanu, Subordinants of differential superordinations, Complex Variables 48(10) (2003), 815-826.

[12] G. Murugusundaramoorthy and N. Magesh, Differential subordinations and superordinations for analytic functions defined by Dziok-Srivastava linear operator, Journal of Inequalities in Pure and Applied Mathematics 7(4) (2006), Article ID 152, 1-20.

[13] V. O. Nechita, Differential subordinations and superordinations for analytic functions defined by the generalized Salagean derivative operator, Acta Univ. Apulensis 16 (2008), 14-156.

[14] D. Răducanu and V. O. Nechita, A differential sandwich theorem for analytic functions defined by the generalized Salagean operator, Aust. J. Math. Anal. Appl. 9(1) (2012), 1-7.

[15] St. Ruscheweyh, New certain for univalent functions, Proc. Amer. Math. Soc. 49 (1975), 109 115.

[16] G. Salagean, Subclasses of univalent functions, in: C. A. Cazacu, N. Boboc, M. Jurchescu and I. Suciu (Eds.), Complex Analysis - Fifth Romanian-Finnish Seminar, Lecture Notes in Mathematics 1013, Springer-Verlag, Berlin, 1983, 362-732.

[17] T. N. Shanmugam, V. Ravichandran and S. Sivasubramanian, Differential sandwich theorems for some subclasses of analytic functions, Aust. J. Math. Anal. Appl. 3(1) (2006), 1-11.

[18] T. N. Shanmugam, C. Ramachandran, M. Darus and S. Sivasubramanian, Differential sandwich theorems for some subclasses of analytic functions involving a linear operator, Acta Math. Univ. Comenianae 74(2) (2007), 287-294.

[19] T. N. Shanmugam, S. Sivasubramanian and H. Silverman, On sandwich theorems for some classes of analytic functions, Int. J. Math. Math. Sci. (2006), Article ID 29684, 1-13.

\author{
${ }^{1}$ Department of Mathematics, \\ College of SCIENCE, \\ UNIVERSITY OF AL-QADISIYAH, \\ DIWANIYA, IRAQ \\ Email address: abbas.kareem.w@qu.edu.iq \\ ${ }^{2}$ Department of Mathematics and Computer Science, \\ UNIVERSITY OF ORADEA, \\ 1 Universitati Street, 410087 Oradea, Romania \\ Email address: dalb@uoradea.ro
}

